\title{
LA SABBIA E I FISICI
}

\author{
Nota del m.s. JACQUES VILLAIN (*)
}

(Adunanza del 25 ottobre 2012)

SuNTO. - Partendo da considerazioni sulla utilizzazione della sabbia come strumento di visualizzazione di alcuni fenomeni, la nota esamina il comportamento dinamico di sistemi granulari sottoposti a sollecitazioni. Si illustrano in particolare le modificazioni da apportare alla descrizione termodinamico-cinetica classica dei gas di molecole in ragione della non-conservazione della energia nelle collisioni tra i grani e le inaspettate conseguenze concettuali e pratiche di tali modificazioni.

$* * *$

ABSTRACT. - The dynamical response of granular systems under certain excitations is considered. In particular the breakdown of the energy conservation in the collisions among grains, with respect to the classical termodynamical- kinetical description of the molecule in gases, is taken into account and it is addressed how unexpected conceptual and pratical consequences arise.

\section{LA SABBIA CHE SI ARRAMPICA}

Da molto tempo la sabbia è stata usata dai fisici come uno strumento. Un impiego di particolare successo fu quello del tedesco Chladni che, due secoli fa, usò la sabbia per evidenziare i modi di vibrazione di una lastra. Le figure di Chladni [1] sono anche utili per mostrare le onde stazionarie in un violino o una chitarra.[2] Invece della sab-

(*) Theory group, ESRF, 6 Rue Jules Horowitz, 3800 Grenoble.

E-mail: jvillain@esrf.fr 
bia, altri materiali granulari possono essere usati, e nella presente comunicazione, la parola "sabbia" deve essere considerata come un'espressione generale per indicare "materia granulare".

Alcuni anni dopo, Michael Faraday cominciò a usare la sabbia come specifico oggetto di studio. Scuotendo uno strato di sabbia inizialmente piano, osservò che si formano mucchi.[3]

Da un quarto di secolo, la sabbia scossa è diventata oggetto di studio abbastanza comune, che ha attratto l'attenzione di alcuni fisici illustri. In questa nota sono ricordate alcune proprietà legate a questo problema e fatte alcune osservazioni ad integrazione.

Si può dapprima ricordare un lavoro di Bizon et al. [4] che, scuotendo uno strato di sabbia, hanno osservato la formazione di una grande varietà di figure, molto più complicate dei mucchi di Faraday. Infatti la sabbia viene proiettata nell'aria, dove i grani subiscono collisioni, poi ricadono sulla lastra (Douady et al. Europhys. Lett., 8, 621, 1989). Durante ogni periodo, la sabbia è ora nell'aria, ora sulla lastra, e la sua posizione sulla lastra cambia ad ogni periodo.

In un altro tipo di esperimenti, la sabbia è messa in una scatola scossa con violenza, e la maggioranza dei grani rimane quasi sempre nell'aria. Parlerò più in dettaglio di questa situazione perché il gas formato dalla sabbia ha proprietà sorprendenti.

\section{LA SABBIA IN VOLO}

Consideriamo una scatola in forma di parallelepipedo, scossa nella direzione verticale $z$, e molto grande nelle direzioni orizzontali. La quantità caratteristiche sono soltanto funzioni di $z$. Il caso più semplice è quello senza gravità, studiato sperimentalmente per esempio da Evesque.[5] (Fig. 1)

I grani di sabbia (che in realtà sono generalmente sfere identiche di massa $m$ ) formano un gas, le cui particelle collidono l'un l'altra. Le collisioni conservano l'impulso, ma non conservano l'energia cinetica! In ogni collisione una parte dell'energia cinetica è trasformata in calore, e questo calore è in qualche modo dissipato e può essere dimenticato. La perdita di energia in ogni urto può essere dell'ordine del $10 \%$. La velocità delle particelle quindi diminuisce quando aumenta la distanza alle pareti vibranti.

Il caso più interessante è il regime stazionario. In questo caso 
abbiamo due ugualianze utili. In primo luogo, il flusso di particelle deve essere nullo, cioè, se $\rho\left(z, \mathrm{v}_{z}\right)$ è la densità locale di particelle di velocità $\mathrm{v}_{z}$, avremo

$$
\int_{-\infty}^{\infty} v_{z} \rho\left(z, v_{z}\right) d v_{z}=0
$$

Un'altra particolarità del caso stazionario è che lo sforzo $\sigma$ deve essere uniforme. La componente più interessante è $\sigma_{z z}$, che chiamerò $P$. È facile dimostrare [6] che, se $\rho$ è la massa per unità di volume, si ha

$P=\sigma_{z z}=\rho<\mathrm{v}_{\mathrm{z}}^{2}>$

dove $<\ldots$. è il valore medio e $v$ è la velocità delle particelle. Questa relazione è del tutto analoga a quella di un gas perfetto all'equilibrio, $P V=N k_{B} T$, ma la temperatura locale $T$ definita da $m\left\langle\mathrm{v}_{\mathrm{z}}^{2}\right\rangle=k_{B} T$ dipende da $z$ e, come abbiamo visto, diminuisce quando aumenta la distanza dalle pareti vibranti. Siccome la pressione (2) è uniforme, la densità $\rho$ aumenta con questa distanza.

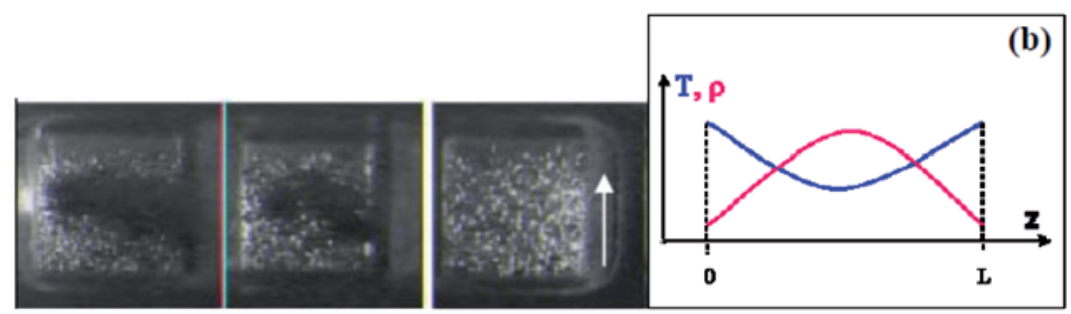

Fig. 1 - Contenitore vibrante con (dalla sinistra alla destra) 3, 2 et 1 strati di palline (Evesque 2009). La zona centrale scura è una zona da alta densità.

A destra, variazione della densità $\rho$ (in rosso) e dell'energia cinetica media $\mathrm{T}$ (in blu) in una scatola di altezza $\mathrm{L}$ in gravità nulla.[5]

Se lo sforzo può facilmente essere definito nel caso stazionario, sarebbe difficile dare una tale definizione fuori dall'equilibrio....

Evesque ha introdotto uno sforzo asimmetrico:

$P^{+}(z)=\sigma_{z z}{ }^{+}=\rho<v_{z}^{2}>^{+}=\int_{0}^{\infty} v_{z}^{2} \rho\left(z, v_{z}\right) d v_{z} \quad: \quad P(z)=\sigma_{z z}{ }^{-}=\rho<v_{z}^{2}>^{-}=\int_{-\infty}^{0} v_{z}^{2} \rho\left(z, v_{z}\right) d v_{z}$ (3) 
Queste quantità non sono uguali, particolarmente nella vicinanza di una parete vibrante. Consideriamo la parete inferiore. Le particelle da velocità positiva sono molto più veloci delle particelle a componente della velocità negativa, che hanno perso energia in seguito a collisioni. Ma la (1) impone che il prodotto $\left.\rho\right|_{z} \mid$ sia uguale in valore medio per $i$ due tipi di particelle. Il prodotto $\left.\left.\rho\right|_{\mathrm{z}}\right|^{2}$ deve quindi essere maggiore per le particelle a velocità positiva, e $P^{+}>P^{-}$. Vicino alla parete superiore $P^{+}<P^{-}$, e al centro, $P^{+}=P^{-}$.

L'interesse di $P^{+}$e $P^{-}$è che la loro differenza ha un significato fisico anche fuori dalla stazionarità. Infatti, se mettiamo nel sistema una particella pesante, la forza che agisce su questa particella è

$F=k[P+(z)-P-(z)]$

che non è nulla, tranne al centro della scatola. Il coefficiente $k$ è dell'ordine di grandezza della sezione della particella, cioè $\pi R^{2}$ per una sfera di raggio $R$. La particella pesante è spinta verso il centro della scatola.

\section{Segregazione E Diavoletto di MaXwell}

Delle osservazioni precedenti si deduce che, nell'assenza di gravità, particelle pesanti vengono attratte dalla parte media della scatola. Questo è un caso di segregazione frequentemente osservato nella sabbia scossa. Un altro esempio classico è quello di una miscela densa di grani grossi e piccoli. Se la miscela è scossa durante un tempo abbastanza lungo, le particelle più grandi salgono alla superficie (Fig. 2). Il fenomeno è differente di quello che abbiamo descritto, e a nostra conoscenza non è ancora del tutto compreso.
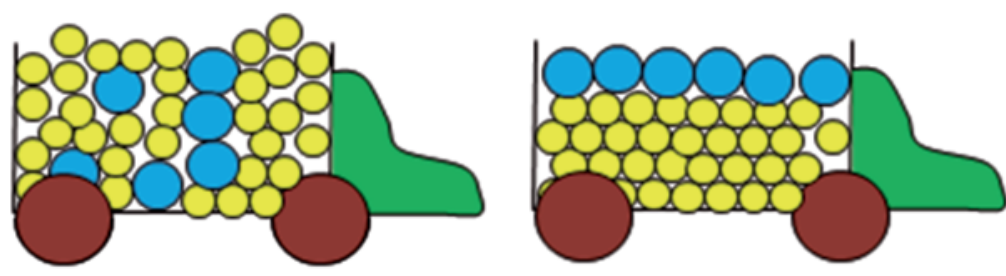

Fig. 2 - Un camion, partendo con un carico di noci mescolate, arriva con le noci più grandi sullo strato superiore. 
Un caso più strano di segregazione può essere osservato scuotendo una scatola a due compartimenti (Fig. 3). In certi casi un compartimento si svuota in parte, e nello stato finale i due compartimenti contengono un numero disuguale di particelle. Questo fatto, degno di un "diavoletto di Maxwell", dimostra la caduta di validità delle proprietà generali della meccanica statistica usuale, che sono il principio del bilancio dettagliato, il principio di Carnot e il teorema H. L'effetto è stato osservato sperimentalmente in presenza di gravità $[7,8]$ se la scatola è scossa con un'energia sufficiente, e senza gravità [9] è stato ottenuto in simulazioni quando il numero di particelle è abbastanza grande.

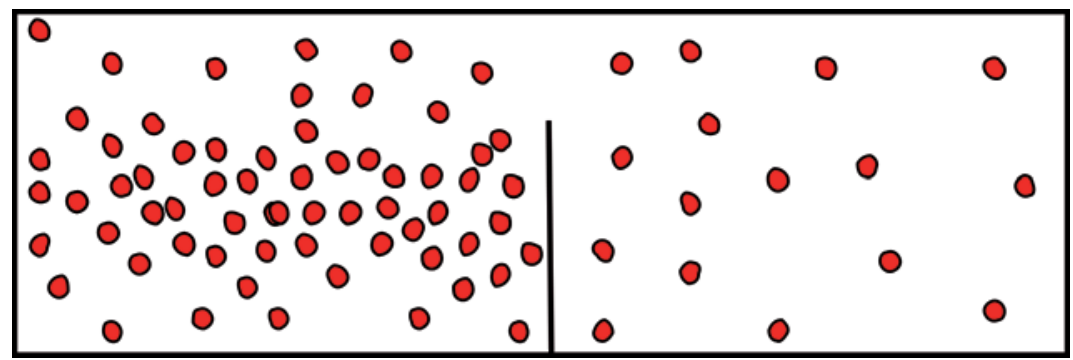

Fig. 3 - Il diavoletto di Maxwell. In certi casi la situazione stabile corrisponde a densità diverse in due compartimenti comunicanti.

Meno strano, ma interessante, è il fatto (Fig. 4) che una clessidra si svuota più lentamente quando viene scossa.[10] Un nuovo diavoletto impedisce alla gravità di operare. Un modo di spiegarlo è di dire che la densità è più bassa nella vicinanza della parete, e quindi della buca. Non lasciamo la clessidra senza osservare che, se non la scuotiamo, la portata è costante, indipendente dell'altezza di sabbia. Questa proprietà è caratteristica della materia granulare.
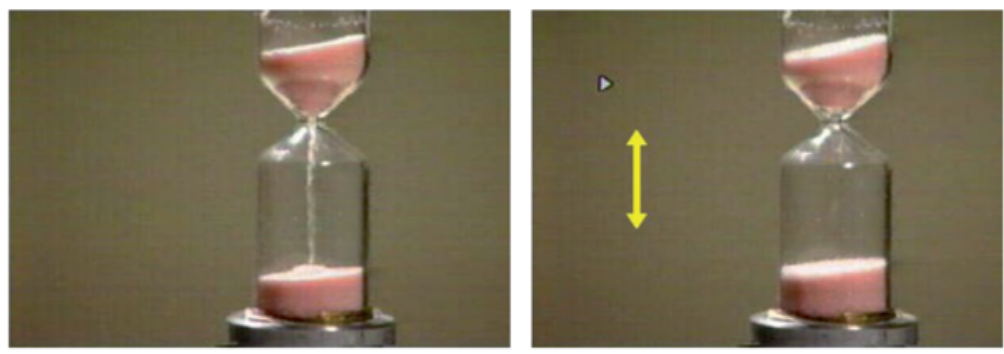

Fig. 4 - Una clessidra a riposo (a sinistra) si svuota normalmente, quando viene scossa quasi smette di svuotarsi (a destra). 


\section{TRACCE SULLA SABBIA}

In un loro libro, Rigamonti e Varlamov [11] riportano le seguenti parole di Reynolds, il grande esperto di idrodinamica, scritte nel 1885: "Quando il piede preme sulla sabbia, sul tratto compatto dopo il ritiro della marea, la regione che si trova attorno al piede diventa immediatamente asciutta. La pressione del piede rende la sabbia meno compatta e quanto più tale pressione è forte, tanta più acqua fuoriesce... Essa rende la sabbia asciutta finché dal basso non arriva altra acqua".

Se capisco bene, questo significa che dopo il ritiro della marea la sabbia ha trovato una struttura ben compatta che lo sforzo verticale del piede perturba, così formando lacune dove l'acqua può penetrare in maggiore quantità (Fig. 5). È usuale, particolarmente in elasticità, che una compressione in una direzione produca un'espansione nelle alte, ma generalmente il volume diminuisce. In elasticità, non credo che sia conosciuto un caso dove la compressione in una direzione produca un aumento di volume. Per la sabbia (che ovviamente non è mezzo elastico) è così.
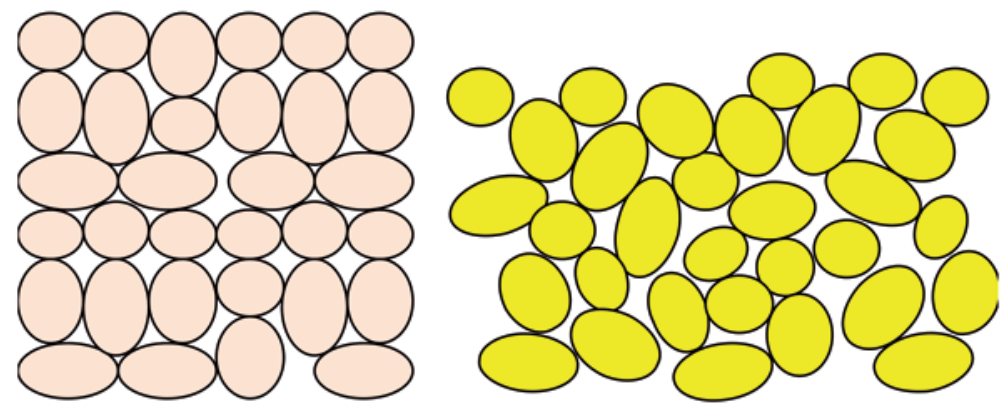

Fig. 5 - Una sabbia che ha formato una struttura ben compatta (a sinistra) prende una forma meno compatta sotto l'effetto di uno sforzo.

Il lettore troverà altri aspetti sulla sabbia nel libro citato, che presenta versioni in inglese e in russo. Una versione innovata ed arricchita è disponibile in francese, con aggiornamenti e integrazioni.[12] Ma ci sono libri interi sulla fisica della sabbia, dei quali la presente nota è soltanto una parziale illustrazione. $[13,14]$ Per quanto riguarda in particolare l'effetto dello sforzo sulla densità, si può utilmente ricorrere all'articolo di rassegna di Evesque.[15]

Per finire voglio ricordare, citando de Gennes,[16] che la mecca- 
nica della sabbia non è soltanto importante dal punto di vista dei fondamenti della fisica, è anche importante per la sopravvivenza degli uomini che vivono intorno al deserto.

\section{BIBLIOGRAFIA}

[1] Ernst F.F. Chladni, Entdeckungen über die Theorie des Klange (1787).

[2] G. Benedetto, R. Pisani, Articolo Acustica musicale (Tavola V) Enciclopedia delle scienze fisiche, Treccani, Roma (1992).

[3] M. Faraday, Philos. Trans. R. Soc. London 52, 299 (1831).

[4] C. Bizon, M.D. Shattuck, J.B. Swift, W.D. McCormick, and Harry L. Swinney., Physical Review Letters 80, 57 (1998).

[5] P. Evesque, Poudres et grains 17, 577 (2009).

[6] J. Villain, Poudres \& grains 20, 29 (2012).

[7] J. Eggers, Phys. Rev. Lett. 83, 5322 (1999).

[8] Una bella realizzazione sperimentale del diavoletto di Maxwell, fra altri esperimenti sulla materia granulare, è visibile sul sito http://www.palais-decouverte.fr/ index.php?id $=1662$

[9] Y. Li, M. Hou, P. Evesque, Journal of Physics: Conference Series 327 (2011) 01203410.

[10] P. Evesque \& W. Meftah, C.R. Acad. Sciences 314, série II, 1125-32, (1992).

[11] A. Rigamonti, A. Varlamov, Magico caleidoscopio della fisica. La Goliardica pavese (2007).

[12] A. Rigamonti, A. Varlamov, J. Villain, Le kaléidoscope de la Physique, Ed. Belin (Paris 2014).

[13] Jacques Duran Poudres, sables et grains (Eyrolles, 1997) Sand, powders and grains (Springer 2000) http://books.google.fr/books?id=9kK1ZxsAS_EC\&printsec= frontcover\&hl=fr\# $\mathrm{v}=$ onepage $\& \mathrm{q} \& \mathrm{f}=$ false

[14] Jacques Duran Sables émouvants (Belin, 2003).

[15] P. Evesque, Eléments de mécanique quasi-statique des milieux granulaires mouillés ou secs, Poudres \& grains NS 1, 1-60 (2000). http://www.poudres-etgrains.ecp.fr/IMG/pdf/poudresNS1.pdf

[16] http://archives.arte.tv/hebdo/archimed/19980915/ftext/sujet5.html 
